\title{
Soft Computing Methods in Business Optimization
}

\section{Petr Dostal}

Department of Business and Management, Brno University of Technology, Czech Republic

\section{Introduction}

There are some tasks that nature manages to perform very easily but which algorithms designed by human beings cannot complete. We can find these tasks in complicated and variable environments. Mathematicians have turned their attention to nature, and on the basis of analogy they created the fuzzy logic, neural networks, and evolutionary algorithms theories. There are various areas of use, such as optimization in management, risk management, decision making, the stock market, technology control, simulation, prediction, cluster analyses, and other branches of applications in business.

The use of the theories mentioned above is in the sphere of optimization. We can generally mention, for example, an optimization of technological decision-making processes with the aim of optimization (minimum losses and expenses, maximum profit), an optimization of capital decision making, an optimization of portfolios, an optimization of prices of products and volume of production, a solution of the problems of travel of salesmen/saleswomen, etc. The business systems belong to the most complicated dynamic systems.

The applications in business have specific features in comparison with applications in engineering. The processes are focused on optimizing income or profit, or on optimizing decreasing expenses. Therefore, such applications, both successful and unsuccessful, are not published very often because of secrecy in the highly competitive environments among firms and institutions. The processes are focused on private corporate attempts at money making. The soft computing methods help in business to target on the right customers and thus it can lead to higher profits and to success in the competitive fight.

There are various soft computing methods used in business: classical ones and methods using soft computing. The decision-making processes in business are very complicated, where many variables are difficult to measure; they are characterized by imprecision, uncertainty, vagueness, semi-truth, approximation, non-linearity etc. Under these conditions, the methods of soft computing such as fuzzy logic, neural networks, evolutionary algorithms are appropriate. Generally speaking, it could be said that the field of applications of soft computing methods in business covers a wide area of applications.

\section{History}

Fuzzy logic differs from conventional (hard) computing in that, unlike hard computing, it is tolerant of imprecision, uncertainty, partial truth, and approximation to achieve tractability, robustness and low solution cost. In effect, the role model for fuzzy logic is the human mind. The basic ideas underlying soft computing in its current incarnation have links to many earlier influences, among them Zadeh's 1965 paper on fuzzy sets [1]; the 1973 paper on the analysis of complex systems and decision processes; and the 1979 report on possibility theory and soft data analysis.

The invention of neural computing came at a later point.The invention of biological neuron was done by Purkinje [2]. The history of the development of neural networks started in the first half of the twentieth century. The first publications were introduced by McCulloch.
Later Pitts worked on the simplest model of a neuron, and after that Rosenblatt created a functional perception that solves only problems involving areas that are linearly separable [3]. When the multilayer network was discovered by Rumelhart, then Hinton and Williams created back-propagation methods for multi-layer networks [4]. A great boom of neural network applications has been on going since the mid-1970s.

The invention of evolutionary computing came at a later point. At first genetic processes in nature were discovered in the nineteenth century by Mendel [5] and developed by Darwin [6]. The computer realization of genetic algorithms discovered in the 1970s is connected with the names of Holland [7] and Goldberg [8]. There are various optimization methods that could be used in business, especially when optimization tasks are solved, such as cultural algorithms, a bacteriologic algorithm, an altruism algorithm, an artificial bee colony algorithm, a firefly algorithm, a glowworm swarm algorithm, bat algorithm, a cuckoo algorithm, a krill heard algorithm, a gravitational search algorithm, an intelligent water drops algorithm, a river formation dynamics, a magnetic optimization algorithm, a stochastic diffusion search, a simulated annealing, an exhaustive search, a backtracking algorithm, a hill climbing, a greedy algorithm, a taboo search and others.

At this juncture, the principal constituents of soft computing are fuzzy logic, neural computing, evolutionary computation, machine learning and probabilistic reasoning, with the latter subsuming belief networks, chaos theory and parts of learning theory. What is important to note is that soft computing is not a melange. Rather, it is a partnership in which each of the partners contributes a distinct methodology for addressing problems in its domain. In this perspective, the principal constituent methodologies in soft computing are complementary rather than competitive. Furthermore, soft computing may be viewed as a foundation component for the emerging field of conceptual intelligence [9].

\section{Soft Computing Methods}

In classical logic, a theory defines a set as a collection having certain definite properties. Any element belongs to the set or not according to clear-cut rules; membership in the set has only the two values 0 or 1 . Later, the theory of fuzzy logic was created by Zadeh [1]. Fuzzy logic defines a variable degree to which an element $x$ belongs to the set. The degree of membership in the set is denoted $\mathrm{f}(\mathrm{x})$; it can take on any value

${ }^{*}$ Corresponding author: Petr Dostal, Department of Business and Management, Brno University of Technology, Czech Republic, E-mail: dostal@fbm.vutbr.cz

Received August 15, 2013; Accepted August 16, 2013; Published August 22, 2013

Citation: Dostal P (2013) Soft Computing Methods in Business Optimization Global J Technol Optim 4: e103. doi: 10.4172/1410-3217.1000e103

Copyright: (c) 2013 Dostal P. This is an open-access article distributed under the terms of the Creative Commons Attribution License, which permits unrestricted use, distribution, and reproduction in any medium, provided the original author and source are credited. 
in the range from 1 to 0 , where 0 means absolute non-membership and 1 full membership. The use of degrees of membership corresponds better to what happens in the world of our experience. Fuzzy logic measures the certainty or uncertainty of how much the element belongs to the set. People make analogous decisions in the fields of mental and physical behaviour. By means of fuzzy logic, it is possible to find the solution of a given task better than by classical methods from the point of optimization.

The neural network model represents the thinking of the human brains. The model is described as a "black box." It is not possible to know the inside structure of the system in detail. We make only a few suppositions about the inner structure of the system. It is simulated by a "black box" that enables us to describe the behaviour of the system by the function that performs transformation of input and output. It is suitable to use neural networks in cases where the influences on searched phenomena are random and deterministic relations are very complicated. In these cases we are not able to separate and analytically identify them. They are suitable for optimization of complicated and often irreversible strategic decision making.

The most used and the best evolutionary algorithm is the genetic one. It is used quite often in business optimization. Let us mention a few terms that are used in the branch of genetics: chromosomes, a selection, a crossover, a mutation, a population, parents, and an offspring. The chromosomes consist of genes (bits). Every gene inherits one or several bits and its position in chromosomes. We say that the chromosomes have locus. The information coded in chromosomes consists of phenotypes. Most of the implementations of genetic algorithm work with the original representation of chromosomes are binary representations: 0 and 1 . A chromosome is represented by a binary string, e.g. 0101 . These binary strings mostly represent coded decimal numbers. The operators of selection, crossover, and mutation are most often used in genetic algorithms. The diagram is then chained, where the permitted symbol occurs in at least one position (in the case of binary representation they are 0 or 1). For the handling of chromosomes, several genetic operators have been proposed. The most used operators are a selection, a crossover, and a mutation. The evolutionary algorithms are suitable for complicated optimization tasks.

There are many books focused on the theory and application of soft computing methods for optimization in business, economics, and finance such as [10-12]. The use of soft computing methods in economic and finance generally are mentioned in [13-17]. The use of soft computing in management is mentioned in the books such as [18$20]$ and the use of soft computing in financial forecasting is described in [21-23]

\section{Pros and Cons of Methods}

Fuzzy logic is a theory that uses fuzzy sets and logic. The advantage of fuzzy logic is in the fact that the data for the processing could be imprecision, contradictory, uncertainty, vagueness, semi-truth, approximated data, and so on. The fuzzy logic uses linguistic variables, the rule base or fuzzy sets are easily modified, the input to output are related in linguistic terms, easily understood, a few rules encompass great complexity. It could be mentioned that fuzzy logic model is not a black box, that the rules are clear. The disadvantage of fuzzy logic could be found in the setup of rules of complicated phenomena and that it is requires finer tuning before implementation into practice. The neuro-fuzzy models could be an advantage in case for the setup of rules. The use of fuzzy logic is possible in numerous applications in business leading to optimum profit.
The advantages of the use of neural networks are in the cases where the influences on searched phenomena are random and deterministic relations are very complicated. In these cases we are not able to separate and analytically identify them. They are suitable for simulation of complicated and often irreversible strategic decision making. The disadvantage of the neural network is that it is as a "black box." It is not possible to know the inside structure of the system in detail. We make only a few suppositions about the inner structure of the system. It is simulated by a "black box" that enables us to describe the behaviour of the system by the function that performs transformation of input and output. The use of neural networks is possible in various applications leading to optimum solution.

The evolutionary algorithm enables the solution of optimization of complicated optimization tasks in business. The disadvantage of some evolutionary algorithms to find the global optimum. There are many problems in business that are optimized by various optimization methods.

\section{Future of Soft Computing Methods}

The future research must be directed to build-up the models for complex decision making processes in business. The future trends that are expected from the soft computing technologies, which may satisfy these needs, are as follows: new fuzzy, neural networks, evolutionary algorithms models and their combinations. The future research will be focused in various applications to support decision making in business to be quicker and more precise because processed amount of data are increasing exponentially. More and more decision making will be done by automatic systems without influence of human being. These automatic decision systems must be designed to be robust and avoiding failures.

The development of quick, more precise, part-time or fully automated decision making systems where soft computing methods will be used. They will save time, decrease wrong decisions, avoiding human failures, reduce costs that can lead to higher profit, or decrease expenses in business and they can help to compete successfully. A striking example of a particularly effective combination is what has come to be known as "neuro-fuzzy-evolutionary systems".

The successful applications of soft computing and the rapid growth suggest that the impact of soft computing will be felt increasingly in coming years. Soft computing is likely to play an especially important role in science and engineering, but eventually its influence may extend much farther.

In many ways, soft computing represents a significant paradigm shift in the aims of computing - a shift which reflects the fact that the human mind, unlike present day computers, possesses a remarkable ability to store and process information which is pervasively imprecise, uncertain and lacking in categoricity [9].

\section{Conclusion}

The complementarity of fuzzy logic, neural network and evolutionary algorithms has an important consequence: in many cases a problem can be solved most effectively by using fuzzy logic, neural network and evolutionary algorithms in combination rather than exclusively.The rapid grows in number and variety of applications of soft computing methods together with increasing the number of researchers and institutions using these methods prove about the correct way of using such decision making methods. Thebusiness optimization methods play very important roles in companies because 
they help to reduce costs that can lead to higher profit and they can help to compete successfully, or decrease expenses in institutions. There are many applications in business optimization mentioned in literature.

The use of soft computing methods can lead to higher optimum of business decision-making, but generally in many other areas such engineering, technology, public services etc.

\section{References}

1. Zadeh LA (1965) Fuzzy sets. Information and Control 8: 338-353

2. Purkinje J (1825) Observations and Experiments Investigating the Physiology of Senses, Prague, Czech Republic.

3. McCulloch W, Pitts W (1943) A logical calculus of the ideas immanent in nervous activity. Bulletin of Mathematical Biophysics 7:115-133.

4. Rumelhart D, Hinton G, Williams R (1986) Learning representations by backpropagating errors. Nature $323: 533-536$.

5. Mendel G (1866) Versucheüberpflanzen-hybriden.Verh.Naturforsch. Ver. Brünn, Brno 4: 3-47.

6. Darwin Ch (1859) The origin of specimens by means of natural selection, John Murray: London.

7. Holland J (1992) Adaptation in natural and artificial systems: An introductory analysis with applications to biology, control, and artificial intelligent. MIT Press, USA.

8. Goldberg D (1997) Genetic algorithms in search, optimization, and machine learning, Addison Wesley, USA.

9. Zadeh LA (2012) A Definition of Soft Computing-adapted from LA Zadeh.

10. Dostál P (2012) The use of soft computing for optimization in business, economics, and finance. In Meta-Heuristics Optimization Algorithms in Engineering, Business, Economics, and Finance, IGI Globe, USA.
11. Dostál P (2012) The use of optimization methods in business and public services. Handbook of Optimization, Springer, USA.

12. Vasant $P$ (2003) Application of fuzzy linear programming in production planning, Fuzzy Optimization and Decision Making. 2: 229-241.

13. Chen S, Wang P, Wen $T$ (2004) Computational intelligence in economics and finance. Springer, USA.

14. Chen S, Wang P, Wen T (2007) Computational intelligence in economics and finance. Volume II, Springer, USA.

15. Dostal P (2011) Advanced decision making in business and public services CERM Academic Publishing House, Czech Republic.

16. Ribeiro R, Zimmermann HJ, Yager R (1999) Soft Computing in Financial Engineering, Springer, USA.

17. Altroc C (1996) Fuzzy logic and neurofuzzy-applications in business and finance. Prentice Hall, USA.

18. Dostál P (2013) The use of soft computing methods for forecasting in business their applications in practice. In Nostradamus: Modern Methods of Prediction, Modeling and Analysis of Nonlinear Systems, Springer, USA.

19. Gil-Lafuente AM, Gil-Lafuente J, Merigó-Lindahl JM (2012) Soft computing in management and Business Economics, Springer, USA.

20. Ruan D, Fedrizzi M (2001) Soft Computing for Risk Evaluation and Management, Springer, USA

21. Dostál $P$ (2013) The use of soft computing in management. In: Vasant $P$ (eds) Handbook of Research on Novel Soft Computing Intelligent Algorithms: Theory and Practical Applications, IGI Globe, USA.

22. Gately E (1996) Neural networks for financial forecasting. John Wiley, USA.

23. Azoff EM (1994) Neural network time series forecasting of financial markets. (1stedn), John Wiley, USA. 